\title{
The Overall Distribution Pattern of the Hand's Cutaneous Nerves and Its Clinical Implications in Sensory Reconstruction
}

\author{
Patrón de Distribución General de los Nervios Cutáneos de la Mano \\ y sus Implicancias Clínicas en la Reconstrucción Sensorial
}

Hui Li; Xiangnan Hu \& Shengbo Yang

LI, H.; HU, X. \& YANG, S. The overall distribution pattern of the hand's cutaneous nerves and its clinical implications in sensory reconstruction. Int. J. Morphol., 39(2):447-454, 2021.

SUMMARY: The objective of this study was to reveal the overall distribution pattern of the hand's cutaneous nerves to provide a morphological basis for the selection and matching of the hand skin for sensory reconstruction during flap transplantation. The hands of 12 adult cadavers were used for the study. Palmar region and dorsum of the hand were divided into regions I-VI. The skin of the hand containing subcutaneous fat was removed close to the muscle surface. The modified Sihler's staining method was used to visualize the overall distribution pattern of the cutaneous nerves and the areas they innervate. The median nerve, superficial branch of the ulnar nerve, and the superficial branch of the radial nerve innervated $59.27 \%$ (containing $4.65 \%$ of the palmar cutaneous branch of the median nerve), $36.91 \%$, and $3.82 \%$ of the palm area, respectively. The superficial branch of the radial nerve, the dorsal branch of the ulnar nerve, and the median nerve innervated $45.16 \%, 47.25 \%$, and $7.59 \%$ of the hand's dorsal skin area, respectively. Communication was found between the arborized branches of these cutaneous nerves. Region III of the palm and region VI of the dorsum of the hand had relatively more dense nerve distribution. Except for region V, the density of the total nerve branches in each palm region was higher than that of the dorsum of the hand. The total number of nerve branches in the distal phalanx and dorsum decreased from the thumb to the digitus minimus. Our results provide morphological guidance when designing a reasonable matching flap to improve the hand's sensory function reconstruction.

KEY WORDS: Hand; Cutaneous nerve; Distribution pattern; Sihler's staining; Sensory reconstruction.

\section{INTRODUCTION}

The skin of the hand is innervated by the cutaneous branches of the median, radial, and ulnar nerves (Standring, 2016). There have been many reports on the gross anatomy of the origin, course, main branches, and the scope of these nerves (Park et al., 2010; Choi et al., 2018; Smith \& Ebraheim, 2019). However, the gross anatomy can only visualize the thick nerve branches but cannot show the distribution of the fine nerve branches in areas where they are densely distributed. Histological methods can reveal the density of the fine nerves in the local skin of the hand (Ahcan et al., 2003; Kelly et al., 2005) but cannot show the overall distribution pattern of the nerve branches. Owing to the hand's high sensory demand, detailed research on the distribution of cutaneous nerves could help improve the sensory reconstruction during skin flap transplantation. Sihler's staining method can clearly show the overall intramuscular nerve distribution pattern, a kind of nerve display method from macro to micro (Wang et al., 2020). The modified Sihler's staining method has been successfully used to display the distribution patterns of the occipital and forearm cutaneous nerves (Kwon et al., 2018; Li et al., 2019). This study intends to use the modified Sihler's staining method to display the overall branch distribution pattern of the cutaneous nerves in the hand, further understand the distribution territory of these nerves, and provide a morphological basis for selecting and matching the hand skin for sensory reconstruction during flap transplantation.

\section{MATERIAL AND METHOD}

Specimens and ethics. Twelve Chinese adult cadavers (six men and six women) with a mean age of $60 \pm 7.5$ (range: $35-$ 75) years without skin disease, history of diabetes mellitus, and neurological disease were fixed with formalin. The 
collection and use of specimens were carried out with the approval of the Ethics Commission of the Zunyi Medical University (approval: \#2016-1-006).

Gross anatomy and measurement. According to the definition of the hand in the textbook of anatomy (Drake et al., 2015), the horizontal line between the tip of the ulna and the radial styloid process was taken as the upper boundary, each fingertip as the lower boundary, and the radial lateral edge as the longitudinal incision to remove the skin containing subcutaneous fat close to the muscle surface. The origin, course, thick branches, and innervation types of the cutaneous nerves were observed. Straightedge and Vernier calipers were used to measure the length, width, and thickness of the specimen.

Regions of the hand. Based on the method described by Chen et al. (2005), the palm was partitioned by making horizontal lines through the middle point of the line between the radial side of the distal rasceta and the proximal end of the first thumb palm cross striation (point a), proximal end of the first palm cross striation (point b), midpoint (point c), distal (point d), and intersection of the palmar longitudinal striation and palmar middle striation (point e), thereby dividing the palm into six regions (I, II, III, IV, V, and VI). The intersection points of the horizontal line passing through points c, d, and e were marked as points c', d', and e', respectively, on the radial side of the thumb. The dorsum of the hand was also divided into regions I-VI by the horizontal line of points a, b, c', d', and e' (Fig. 1).

Modified Sihler's staining. After defatting the specimens in absolute ethanol for 3 days, they were hydrolyzed in 0.25
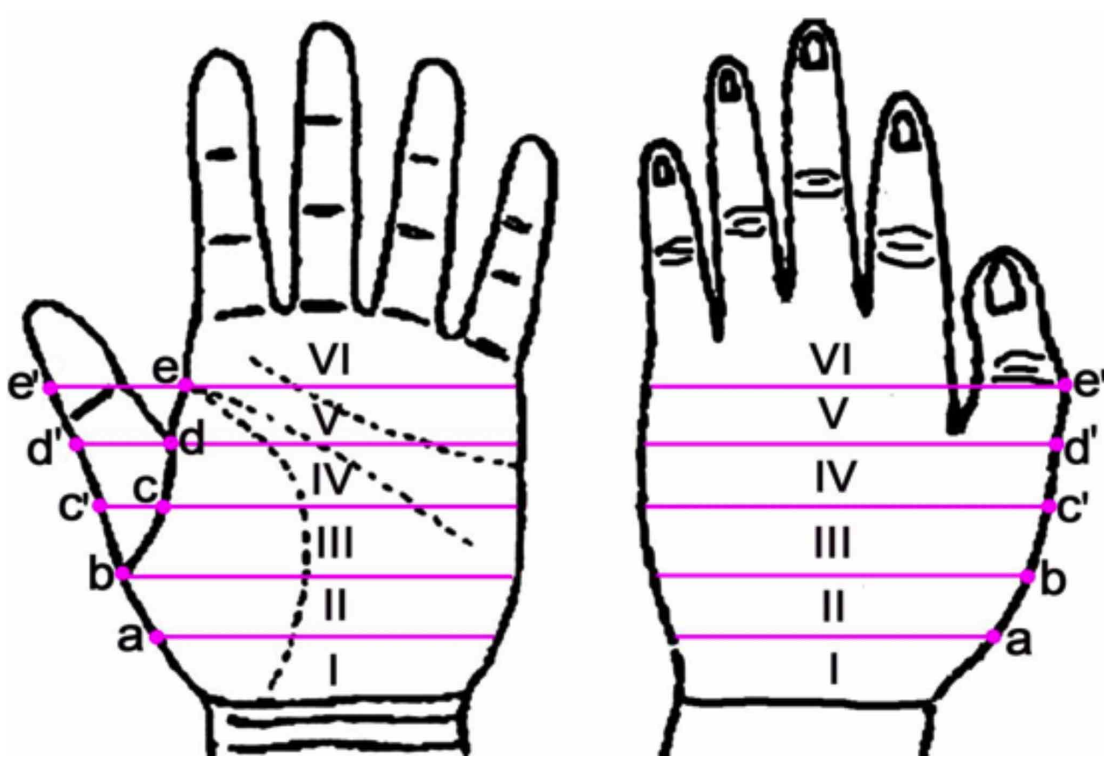

Fig. 1. Schematic drawing of hand division.
$\%$ collagenase for 3 days and then stained using Sihler's method in $0.2 \%$ hydrogen peroxide $+3 \%$ potassium hydroxide solution for 4-5 weeks. They were next treated for 4-5 weeks with Sihler's I solution (one part glacial acetic acid, two parts glycerin, 12 parts $1 \%$ hydrated trichloroacetaldehyde) to decalcify and then with Sihler's II solution (one part Ehrlich hematoxylin solution, two parts glycerin, 12 parts $1 \%$ hydrated trichloroacetaldehyde) for 4 weeks to stain. The specimens were again placed into Sihler's I solution for 2-10h for decolorization, neutralized in $0.05 \%$ lithium carbonate solution for $2 \mathrm{~h}$, and made transparent in gradient glycerol $(40 \%, 60 \%, 80 \%$, and $100 \%)$ for 1 week.

Distribution pattern of the cutaneous nerves. The stained specimens were placed in an X-ray reading box, and the course and distribution of the secondary, tertiary, and following nerve branches were observed visually. After laying a fishing line above the region mark, a ruler was placed in the horizontal and vertical directions next to the specimen; a picture was taken, and a pattern diagram was drawn. Since the specimen shrunk slightly during the decalcification process, the specimen's length and width were remeasured to calculate the scaling coefficient of the specimen area as follows:coefficient $=$ area of the specimen after staining/area of the specimen before staining.

Nerve branch density. Adobe Photoshop 13.0 software (Adobe, CA, USA) was used to determine the nerve branch density. Using the figure's scale value, the vertical and horizontal reference line tools were set to construct a $1 \mathrm{~cm} \mathrm{x} 1$ $\mathrm{cm}$ box. Dragging the reference line from top to bottom and left to right, the density of the secondary, tertiary, and following nerve branches was determined in each region. The density of the nerve branches in a region was calculated as the number of nerve branches in the region/area of the regional specimen $x$ scaling coefficient. Finally, the total density of the nerve branches was calculated by summation.

Statistical analyses. The data were processed using SPSS17.0 software (SPSS Inc, Chicago, Illinois, USA). Oneway ANOVA was used to compare the data from different regions. The GameHowell test was used for pairwise comparison between regions. The paired T-test was used to compare data between the two hands, and the independent sample T-test was used to compare data between men and women, with statistical significance at $P<0.05$. 


\section{RESULTS}

Gross anatomical findings. After the median nerve (MN) divided one palmar cutaneous branch of $\mathrm{MN}(\mathrm{PCBMN})$ on the proximal side of the palm, $54.17 \%(13 / 24)$ and 45.83 $\%(11 / 24)$ of it was divided into two common palmar digital nerves (CPDNs) and three CPDNs, respectively. The superficial branch of the ulnar nerve (SBUN) branched out as a CPDN and a proper palmar digital nerve (PPDN) in the proximal hypothenar. Among the dorsal branch of the ulnar nerve (DBUN), $91.67 \%(22 / 24)$ and $8.33 \%(2 / 24)$ were divided into three and four primary nerve branches, respectively. Among the superficial branch of the radial nerve (SBRN), $91.67 \%(22 / 24)$ and $8.33 \%(2 / 24)$ were divided into three and four primary nerve branches, respectively.

Sihler's staining. After staining, the specimen shrunk slightly, and the overall distribution pattern of the cutaneous nerves of the hand was visible to the naked eye (Figs. 2 to 4). The scaling coefficients of the palm and dorsum of the hand were $0.91 \pm 0.05$ and $0.88 \pm 0.04$, respectively.
Median nerve. The PCBMN separated from the MN was further divided into three branches type $(54.17 \%, 13 / 24)$ and two branches type $(45.83 \%, 11 / 24)$ of the secondary nerve branches. The lateral branch of the three branch type often emitted three tertiary nerve branches, which are distributed in the thenar region (II and III), while the middle and medial branches are distributed in the middle of the palm (regions II-IV) (Figs. 2 and 3). The trunk of the MN branched into an umbrella shape in the third region of the palm. There were two types of branches. Type I accounted for $54.17 \%$ (13/24) of the innervation. The MN branched out into two CPDNs in region III and then divided into seven PPDNs, which proceeded to course within three and a half fingers (index, middle and half the ring finger) along the radial aspect of the hand, forming arborized branches at its terminal point. The branches of the distal finger-pulp were dense and formed rich anastomoses. The PPDN branches near the proximal interphalangeal joint were distributed in the middle and distal phalangeal dorsum of the three and a half fingers on the radial side. Type II accounted for $45.83 \%$ (11/24). The MN branched out into three CPDNs in area III and then divided into seven PPDNs that innervated the three and a half fingers

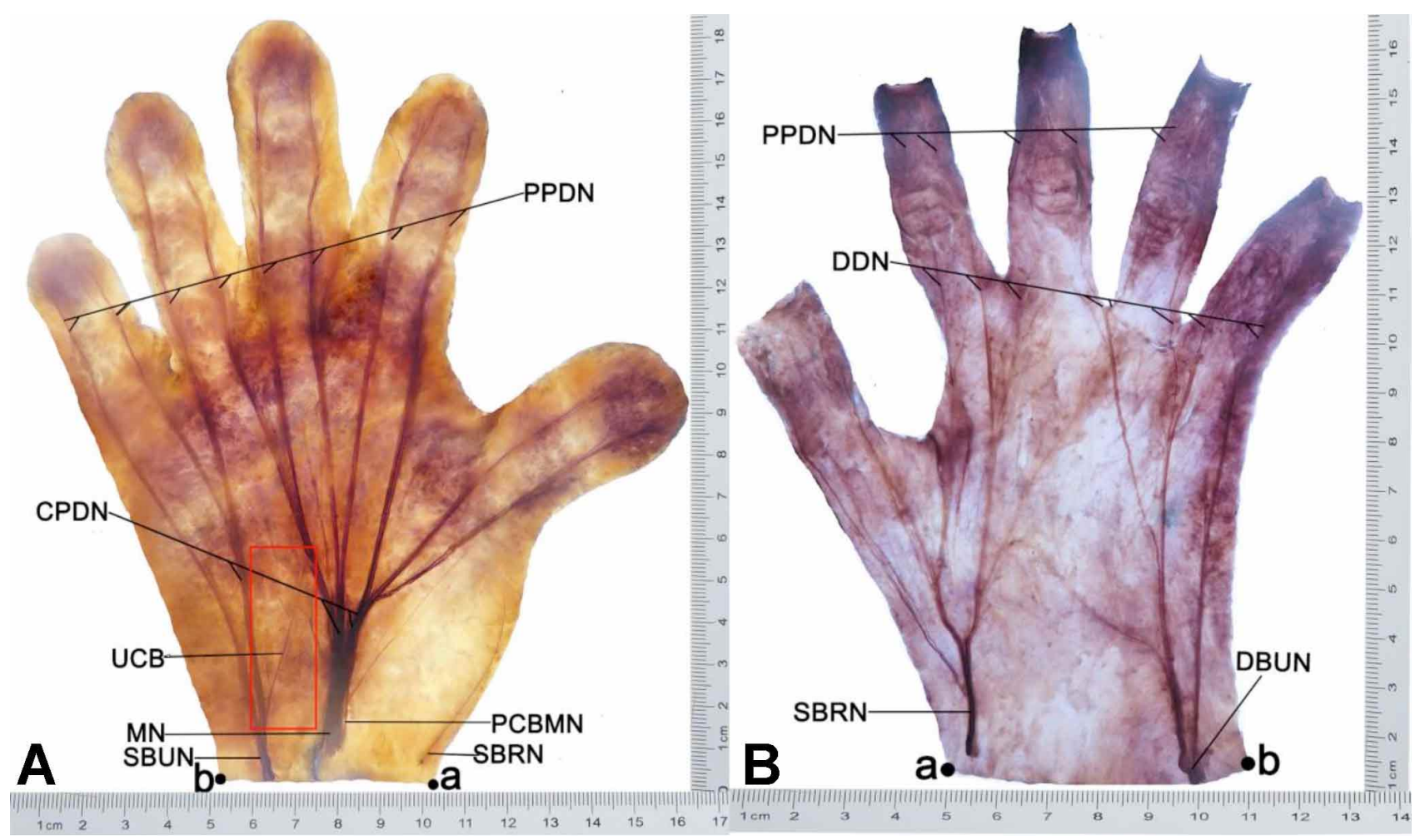

Fig. 2. Sihler's staining showing the distribution of the cutaneous nerves in the right palm and dorsum of the hand. A: palm. $a=$ styloid process tip of radius, $b=$ styloid process tip of ulna; $M N=$ median nerve, $S B U N=$ superficial branch of ulnar nerve, $P C B M N=$ palmar cutaneous branch of median nerve, $\mathrm{SBRN}=$ superficial branch of radial nerve, $\mathrm{CPDN}=$ common palmar digital nerve, $\mathrm{PPDN}=$ palmar proper digital nerve, $\mathrm{UCB}=$ communicating branch of ulnar nerve, red box = communicating branch of the ulnar nerve. B. dorsum of the hand. DBUN = dorsal branch of the ulnar nerve, DDN = dorsal digital nerve. Scale: $\mathrm{cm}$. 
on the radial side. The MN covered $59.27 \pm 7.82 \%$ of the palm surface skin area, of which PCBMN covered $4.65 \pm 0.52$ $\%$ of the palm area. The MN also covered $7.59 \pm 0.86 \%$ of the dorsum of the hand area (Figs. 2 to 4 ).

Superficial branch of the ulnar nerve. The SBUN was first divided into one CPDN and one PPDN in the proximal hypothenar. The CPDN was then further divided into two PPDNs near the fifth metacarpophalangeal joint. The branches of the three PPDNs were distributed to the medial ring finger and the digitus minimus and were especially densely distributed on the distal finger-pulp. In regions II and III, there were communicating branches between the branch of SBUN and CPDN. The SBUN innervated $36.91 \pm 4.67 \%$ of the palm (Figs. 2 to 4 ).

Superficial branch of the radial nerve. In the dorsum of the hand, $91.67 \%(22 / 24)$ of the SBRN in region II was divided into three primary nerve branches, namely the medial, middle, and lateral branches. The medial branch branched out into 4-5 secondary nerve branches to the middle of the dorsum of the hand and communicated with the lateral branch of the ulnar nerve; $8.33 \%(2 / 24)$ of the SBRN were divided into four primary nerve branches. SBRN in the palm divided into the lateral thenar and marginal thumb branches. The SBRN was often distributed to the radial half of the dorsum of the hand. However, on the back of the fingers, it can be divided into three distribution types: type I $(79.17 \%, 19 / 24)$, the nerves were distributed to the dorsum of the proximal thumb and forefinger and to the dorsolateral side of the proximal middle finger; type II $(12.50 \%, 3 / 24)$, the nerves were distributed to the medial dorsum of the proximal thumb, dorsum of the proximal forefinger, and lateral dorsum of the proximal middle finger; type III ( 8.33 $\%, 2 / 24)$, the nerves were distributed to the medial dorsum of the proximal thumb, dorsum of the proximal forefinger, and the lateral dorsum of the proximal middle finger and ring finger. SBRN accounted for $45.16 \pm 4.35 \%$ of the dorsum of the hand skin area and $3.82 \pm 0.47 \%$ of the palm area (Figs. 2 to 4$)$.

Dorsal branch of the ulnar nerve. A major part of the DBUN $(91.67 \%, 22 / 24)$ could be divided into three primary branches in dorsum of the hand region II: medial, middle, and lateral branches. A part of the DBUN $(8.33 \%, 2 / 24)$ was divided into four primary nerve branches. Its distribution can be divided into three types: type I $(87.50 \%, 21 / 24)$, the DBUN was distributed to the dorsum of the proximal digitus minimus and ring fingerand to the medial dorsum of the proximal middle finger; type II $(8.75 \%, 1 / 24)$, the DBUN
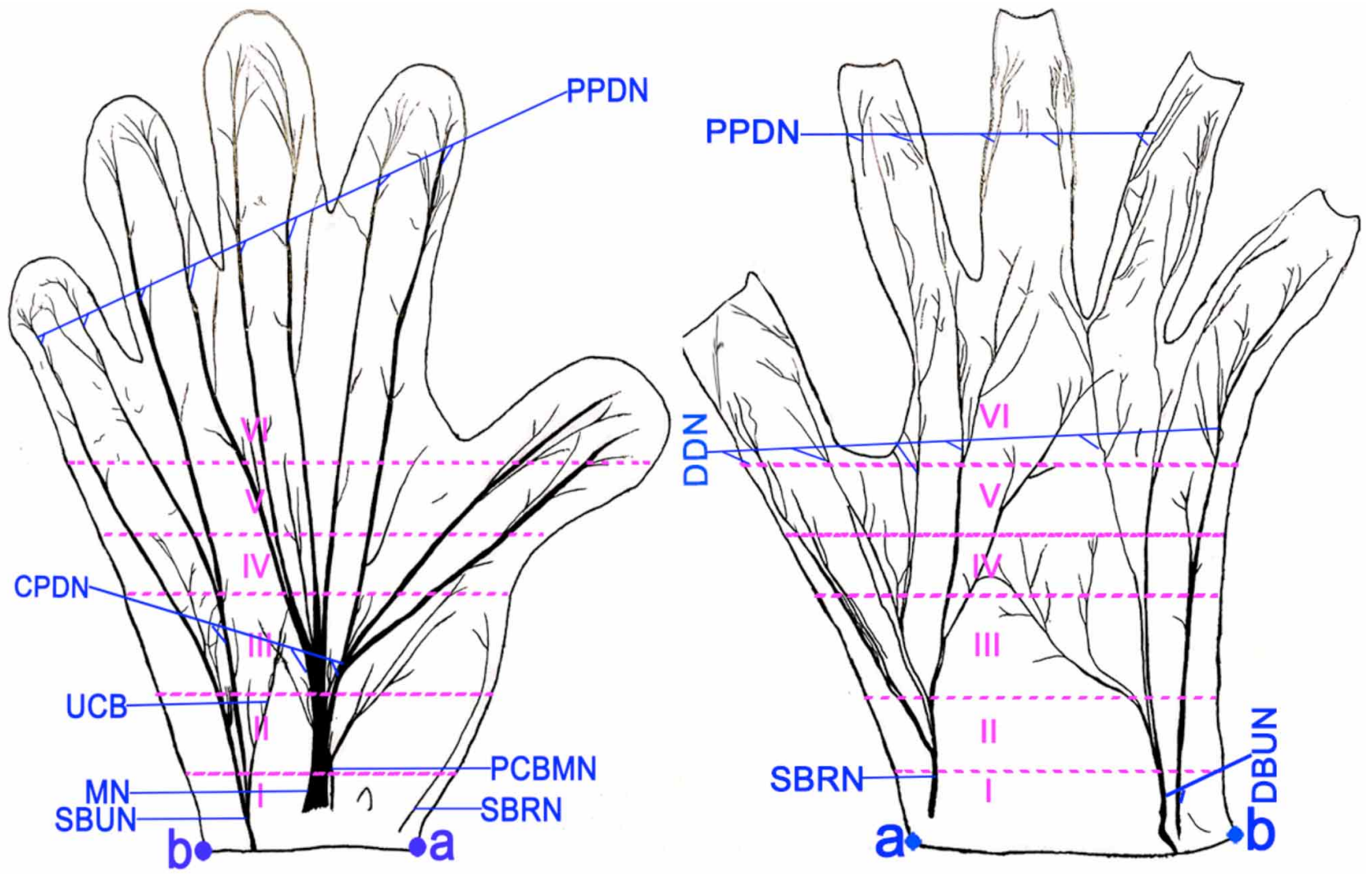

Fig. 3. Schematic drawing of Figure 2 
was distributed to the lateral dorsum of the proximal digitus minimus, dorsum of the proximal index finger, and medial dorsum of the proximal middle finger; and type III $(8.33 \%$, $2 / 24$ ), the DBUN was distributed to the medial dorsum of the proximal digitus minimus, ring finger, and middle finger. The DBUN covered $47.25 \pm 5.27 \%$ of the skin on the dorsum of the hand (Figs. 2 to 4 ).

Thickness, area, and nerve branch density of each region. The thickness, area, and nerve branch density of each region of the palm and dorsum of the hand are shown in Table I. The skin in region IV was the thickest, while in region III was thinnest. Before and after staining, region VI was the largest, and region I was smallest. These differences were significant $(\mathrm{P}<0.01)$. A comparison of the density of secondary nerve branches in each palm region showed that region V had the highest, while region II had the lowest. A comparison of the density of the tertiary and following nerve branches and the total nerve branches in each region of the palm showed that region III had the highest, while region I had the lowest. A comparison of the nerve branch density of the secondary nerve branches between regions I and II; regions III, V, and VI; regions $\mathrm{V}$ and $\mathrm{VI}$, as well as of the tertiary and following nerve branches between regions V, I, and IV and regions I and IV, and a comparison of the total nerve branch density between regions II and IV and regions $\mathrm{V}$ and VI, showed no significant differences $(\mathrm{P}>0.05)$. The comparison between the remaining regions showed significant differences $(\mathrm{P}<0.01)$.

A comparison of the density of secondary nerve branches in each region of the dorsum of the hand showed that regions $\mathrm{V}$ and IV had the highest and lowest, respectively. A comparison of the density of the tertiary and following nerve branches in each region of the dorsum of the hand showed that while region VI had the highest, region II had the lowest. A comparison of the total nerve branch density in each region of the dorsum of the hand showed that regions VI and I had the highest and lowest, respectively. Comparing the secondary nerve branch density between regions III and $\mathrm{V}$ and the tertiary and following nerve branches between regions V and I, regions II and III, and regions I and III showed no significant differences $(\mathrm{P}>0.05)$. A comparison of the total nerve branch density between
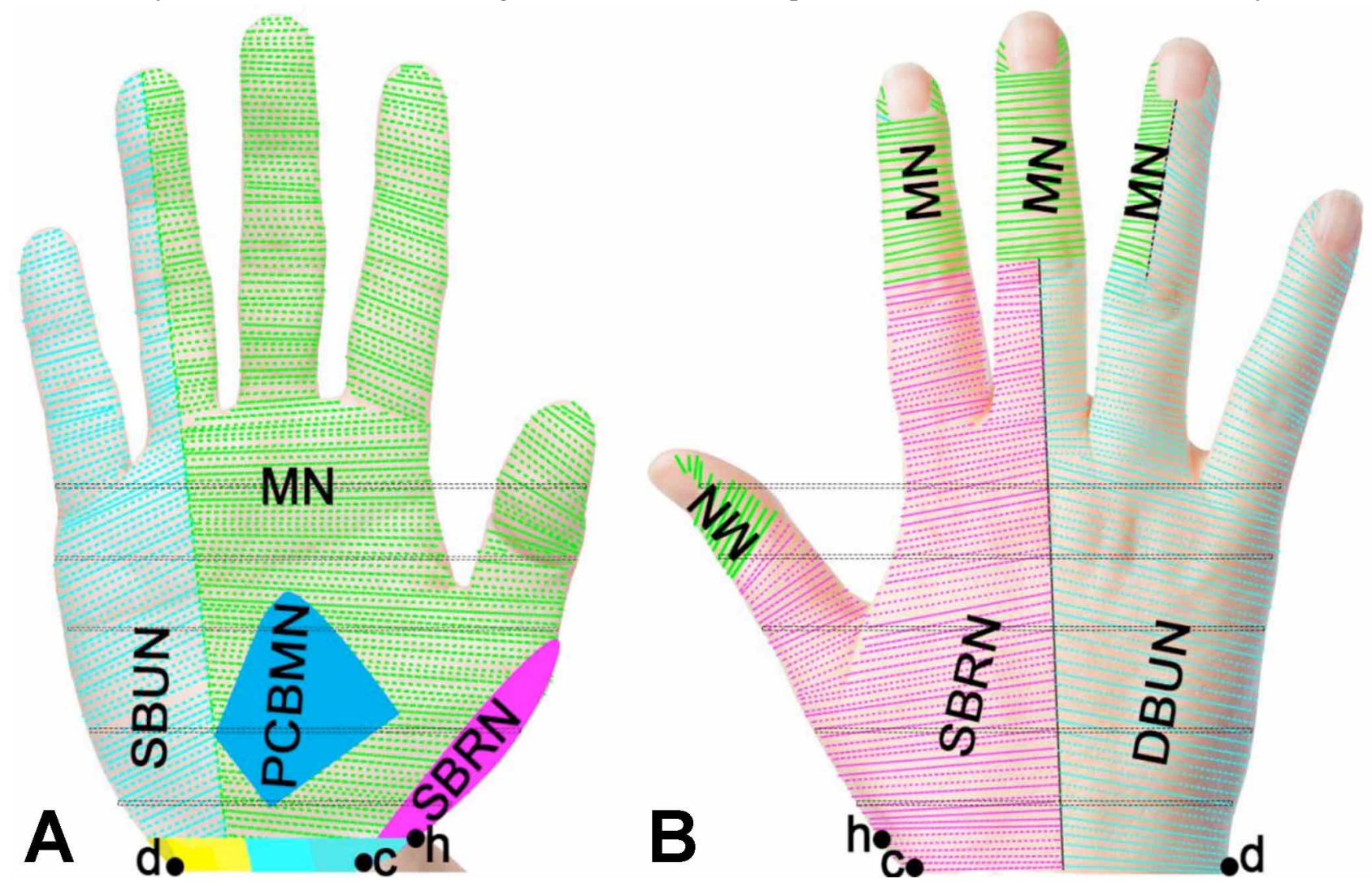

Fig. 4. Distribution of the cutaneous nerves in the right palm and dorsum of the hand. A: Palm. Green = median nerve $(\mathrm{MN})$ distribution area, light blue $=$ superficial branch of ulnar nerve $($ SBUN) distribution area, blue $=$ palmar cutaneous branch of median nerve $(\mathrm{PCBMN})$ distribution area, purple $=$ superficial branch of radial nerve $(\mathrm{SBRN})$ distribution area in the palm area. B. dorsum of the hand. Light blue $=$ dorsal branch of ulnar nerve $(\mathrm{DBUN})$ distribution region, magenta $=$ superficial branch of radial nerve (SBRN) distribution area on the dorsum of the hand. 


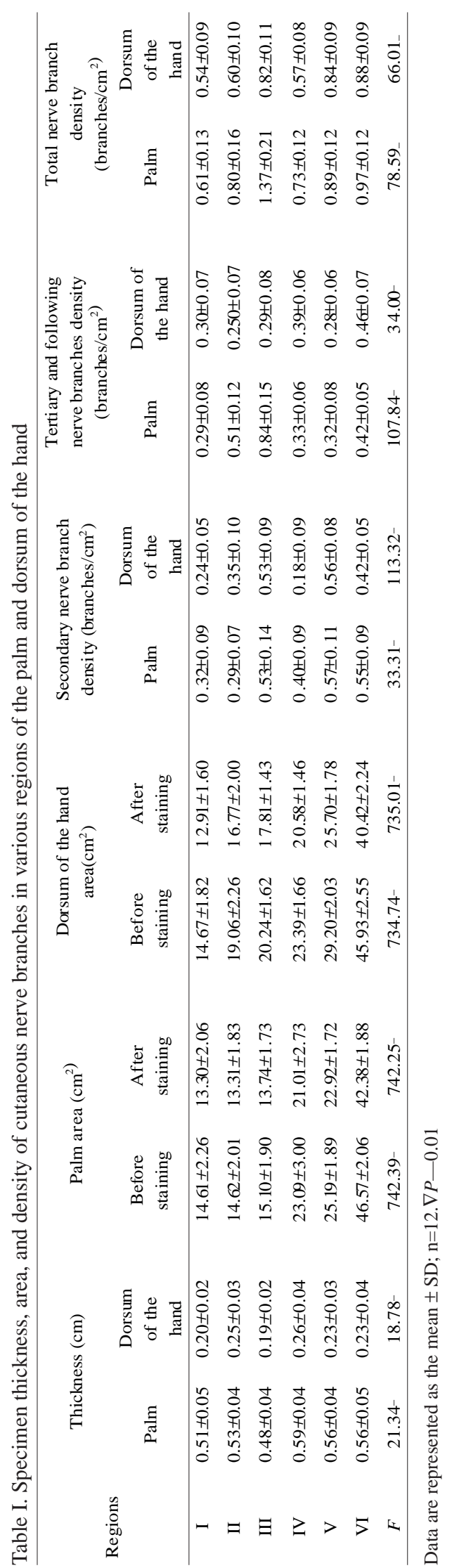

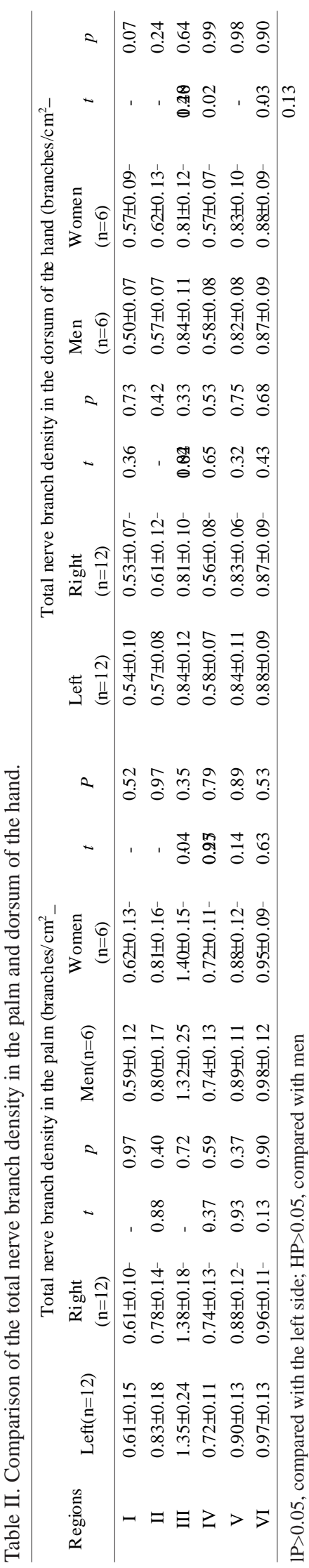

regions IV, I and II, and regions V, III, and VI also showed no significant differences $(\mathrm{P}>0.05)$. The comparison among the remaining regions showed significant differences $(\mathrm{P}<0.01)$. One can see with the naked eye that the nerves distribution of the proximal and middle finger skin was sparser than those distal. The total nerve branch density of the distal fingerpulp in the thumb, index finger, middle finger, ring finger, and digitus minimus was $9.25,8.04,7.08,5.88$, and 4.83 , respectively (decreasing trend).The total nerve branch density of the distal dorsum of the finger in the thumb, index finger, middle finger, ring finger, and digitus minimus was $8.13,6.88,5.92,4.96$, and 3.88 , respectively (decreasing trend). Comparison of the total nerve branch density in the palm and dorsum of the hand between the left and right sides and between men and women showed no significant differences $(\mathrm{P}>0.05)$ (Table II)

\section{DISCUSSION}

For soft tissue defects of the hand, wound repair and sensory function reconstruction are equally important (Bertelli \& Khoury, 1992). Surgeons have applied skin flaps with PCBMN, SBRN, and DBUN to repair the hand's soft tissue defects (Zhang et al., 2015; Usam et al., 2019). This type of skin flap reconstruction can also anastomose the donor and recipient nerves to regain sensation (Patel et al., 2019). However, the sensory recovery of these skin flaps designed based on the known distribution of cutaneous nerves is not ideal. If we can design an area dominated by a dense primary nerve branch or secondary branch as the transplantation object, it can reduce the donor site's sensory defect and meet the reconstruction needs of the sensitive recipient area. If an area 
dominated by a densely branched primary or secondary nerve branches can be designed as the transplant object, it can reduce the donor site's sensory defect and meet the recipient site's demand for sensory acuity reconstruction.Therefore, by further revealing the overall distribution pattern of the hand's cutaneous nerves, this study will help design skin flaps that meet the requirements of sensory function repair and aesthetic reconstruction of the damaged site.

Kanaya et al. (2014) once rotated the neurotrophic flap with the thumb radial palmar proper artery (the artery mainly originating from the main hallux artery) and placed it on the defective thumb. The PCBMN was connected to the thumb under a microscope. The radial finger nerve was anastomosed end-to-end, and the affected area was well innervated after the operation. During the postoperative follow-up, it was found that the dynamic two-point resolution reached $6 \mathrm{~mm}$ and the static two-point resolution reached $10 \mathrm{~mm}$. The PCBMN and PPDN were anastomosed end-toend to repair the soft tissue defect in the finger. The donor site was directly closed and sutured. The postoperative follow-up showed that the skin flap had a good appearance and the distance between the two points was $4.0-7.0 \mathrm{~mm}$. The DBUN is usually sutured with the nerve of the digitus minimus, and it is found that the recovery of the two-point discrimination after nerve anastomosis is better than that without nerve anastomosis (Casoli et al., 2004; Usami et al., 2019). On the one hand, these clinical practices suggest that increasing attention is being paid to the sensory reconstruction and functional recovery of the hand; on the other hand, it shows that performing nerve anastomosis is better than not performing nerve anastomosis. Thus, we aimed to reveal the distribution pattern of cutaneous nerve branches in the hand, which is an essential guide during sensory reconstruction.

However, these flaps were designed for cutaneous nerve trunks and their nutrient vessels without considering the specific details of nerve branches and distribution density. There have been many reports on the distribution of cutaneous nerve branches in the hand. For example, it has been reported that the palmar cutaneous branch of the MN is divided into three nerve branches, namely the lateral, middle, and medial branches (Jones et al., 2016). This is consistent with the results of this study, though we have additionally revealed the two types and the finer secondary and following nerve branches using Sihler's staining (Figs. 2 and 3). Chang \& Cheng (1965) studied six distribution types of dorsal hand cutaneous nerves through gross anatomy, compared them with European and Japanese studies, and reported ethnic differences in specific types. However, they did not reveal that the SBRN described in this study innervated the dorsolateral proximal phalangeal finger. Using Sihler's staining, we observed that $95.83 \%$ of the DBUN and SBRN branches formed communications in the middle of the dorsal hand, which could not be seen by studying the gross anatomy.

We found that the density of the total nerve branches was high in region III of the palm and region VI of the hand's dorsum. Hu et al. (2020) have proposed the principle of "adjacent tissue, good repair and reconstruction of the recipient site, small trauma loss in the donor site" and anastomosis of the donor site with the digital nerve, which can promote the recovery of sensory function after surgery. Based on this principle and the relatively small area of skin for cutting on the palm and the hand's dorsum, which have high requirements for sensory function, our findings suggest that region III of the palm and VI of the dorsum should be the recipient regions with higher sensory needs for sensory reconstruction during skin flap transplantation. When a palmar skin is required as the donor site, it is recommended to design regions IV and $\mathrm{V}$ of the palm because its secondary nerve branches (PPDN) are relatively thick and dense. When a dorsal hand flap transplantation is required, it is recommended that the lateral and medial secondary nerve branches in region III of the dorsal hand for design; however, the central region with the communicating branches of the SBRN and DBUN is not suitable for cutting. The palm has a higher nerve branch density than the dorsum of the hand. The total nerve branches of the distal finger-pulp and the finger's dorsum decreased from the thumb to the digitus minimus, which is related to the need for the palm and thumb to adapt to human labor and to complete fine and complex movements. Notably, no sex-based differences in nerve distribution density were found.

In this study, the modified Sihler's staining method was used to successfully display the overall branch distribution pattern of the hand's cutaneous nerve. We have shown the tiny branches that cannot be revealed by gross anatomy and the nerve communicating locations. We also measured and delineated the density and range of nerve branches, providing a visual anatomical basis for selecting tissues for sensory reconstruction during hand skin flap transplantation.

Our study has some limitations. First is the relatively small sample size. Second, racial differences have not been considered. Finally, each nerve's innervation range was not completely consistent with the boundary depicted in the Netter Atlas (Netter, 2014) and therefore requires multiple verifications. However, this study adds new data on the distribution of the cutaneous nerve branches in the hand, which will help surgeons design a skin flap that can better repair the sensory function in the recipient. 


\section{ACKNOWLEDGEMENTS}

This work was supported by the National Natural Science Foundation of China (No. 31660294, 31540031).

LI, H.; HU, X. \& YANG, S. Patrón de distribución general de los nervios cutáneos de la mano y sus implicancias clínicas en la reconstrucción sensorial. Int. J. Morphol., 39(2):447-454, 2021.

RESUMEN: El objetivo de este estudio fue revelar el patrón de distribución general de los nervios cutáneos de la mano y proporcionar una base morfológica para la selección y adaptación de la piel de la mano, para la reconstrucción sensorial durante el trasplante de colgajo. Para el estudio se utilizaron 12 manos de cadáveres adultos. Las regiones palmar y dorsal se dividieron en regiones I-VI. La piel de la mano que contiene grasa subcutánea se eliminó cerca de la superficie del músculo. Para visualizar el patrón de distribución general de los nervios cutáneos y las áreas que inervan se utilizó el método de tinción de Sihler modificado. El nervio mediano, la rama superficial del nervio ulnar y la rama superficial del nervio radial inervaban el 59,27 \% (que contenía el $4,65 \%$ de la rama cutánea palmar del nervio mediano), el 36,91\% y el 3,82 \% del área de la palma, respectivamente. La rama superficial del nervio radial, la rama dorsal del nervio ulnar y el nervio mediano inervaban el $45,16 \%$, el $47,25 \%$ y el 7,59 \% del área dorsal de la mano, respectivamente. Se observó comunicación entre las ramas arborizadas de estos nervios cutáneos. La región III de la palma y la región VI del dorso de la mano tenían una distribución nerviosa relativamente más densa. A excepción de la región $\mathrm{V}$, la densidad de las ramas nerviosas totales en cada región de la palma fue mayor que el dorso de la mano. El número total de ramas nerviosas en la falange distal y el dorso disminuyó desde el pulgar hasta el dedo mínimo. Nuestros resultados proporcionan una guía morfológica al diseñar un colgajo compatible razonable para mejorar la reconstrucción de la función sensorial de la mano.

PALABRAS CLAVE: Mano; Nervio cutáneo; Patrón de distribución; Tinción de Sihler; Reconstrucción sensorial.

\section{REFERENCES}

Ahcan, U.; Arnez, Z. M.; Bajrovic', F. F.; Hvala, A. \& Zorman, P. Nerve fibre composition of the palmar cutaneous branch of the median nerve and clinical implications. Br. J. Plast. Surg., 56(8):791-6, 2003.

Bertelli, J. A. \& Khoury, Z. Neurocutaneous island flaps in the hand: anatomical basis and preliminary results. Br. J. Plast. Surg., 45(8):58690, 1992.

Casoli, V.; Vérolino, P.; Pélissier, P.; Kostopoulos, E.; Caix, P.; Delmas, V.; Martin, D. \& Baudet, J. The retrograde neurocutaneous island flap of the dorsal branch of the ulnar nerve: anatomical basis and clinical application. Surg. Radiol, Anat., 26(1):8-13, 2004.

Chang, C. M. \& Cheng, G. L. Distribution of cutaneous nerves of hands in Chinese subjects. Acta Anat. Sin., 8(1): 90-7, 1965

Chen, L. F.; Rao, L. B. \& Su, X. L. Division of palm regarding median nerve and ulnar nerve. Chin. J. Anat., 28(1):84-6, 2005.
Choi, P. J.; Nwaogbe, C.; Iwanaga, J.; Georgiev, G. P.; Oskouian, R. J. \& Tubbs, R. S. The deep fascia of the forearm and the ulnar nerve: an anatomical study. Cureus, 10(6):e2842, 2018.

Drake, R. L.; Vogl, A. W. \& Mitchell, A. W. M. Gray's Anatomy for Students. $3^{\text {rd }}$ ed. Philadelphia, Churchill Livingstone, 2015. pp.792-818.

Hu, D.; Wei, Z.; Wang, T.; Hong, X.; Zheng, H. \& Lin, J. Anatomical basis and clinical application of the dorsal perforator flap based on the palmar artery in the first web. Surg. Radiol. Anat., 42(3):269-76, 2020.

Jones, C.; Beredjiklian, P.; Matzon, J. L.; Kim, N. \& Lutsky, K. Incidence of an anomalous course of the palmar cutaneous branch of the median nerve during volar plate fixation of distal radius fractures. J. Hand Surg. Am., 41(8):841-4, 2016

Kanaya, K; Wada, T; Iba, K. \& Yamashita, T. Innervated thenar pedicle flap with the palmar cutaneous branch of the median nerve for treating a thumb pulp defect: a case report. J. Reconstr. Microsurg., 30(5):363-6, 2014.

Kelly, E. J.; Terenghi, G.; Hazari, A. \& Wiberg, M. Nerve fibre and sensory end organ density in the epidermis and papillary dermis of the human hand. Br. J. Plast. Surg., 58(6):774-9, 2005.

Kwon, H. J.; Kim, H. S.; O, J.; Kang, H. J.; Won, J. Y.; Yang, H. M.; Kim, S. H, \& Choi, Y. J. Anatomical analysis of the distribution patterns of occipital cutaneous nerves and the clinical implications for pain management. $J$. Pain Res., 11:2023-31, 2018.

Li, H.; Zhu, W.; Wu, S.; Wei, Z. \& Yang, S. Anatomical analysis of antebrachial cutaneous nerve distribution pattern and its clinical implications for sensory reconstruction. PloS One, 14(9):e0222335, 2019.

Netter, F. H. Atlas of Human Anatomy. $6^{\text {th }}$ ed. Philadelphia, Saunders Elsevier, 2014. pp.44.

Park, B. K.; Bun, H. R.; Hwang, M.; Hong, J. \& Kim, D. H. Medial and lateral branches of the superficial radial nerve: cadaver and nerve conduction studies. Clin. Neurophysiol., 121(2):228-32, 2010.

Patel, K. B.; Naik, A. N.; Kang, S. Y. \& Old, M. O. Aberrant superficial branch of the radial nerve-radial forearm free flap considerations. Plast. Reconstr. Surg. Glob. Open, 7(6):e2191, 2019.

Smith, J. L. \& Ebraheim, N. A. Anatomy of the palmar cutaneous branch of the median nerve: A review. J. Orthop., 16(6):576-9, 2019

Standring, S. Gray's Anatomy. The Anatomical Basis of Clinical Practice. Singapore, Churchill Livingstone, 2016. pp.783-6.

Usami, S.; Kawahara, S.; Inami, K. \& Hirase, Y. Use of a vascularized dorsal sensory branch of an ulnar nerve flap for repairing a proper digital nerve with coverage of a volar soft tissue defect: Report of two cases. Microsurgery, 39(7):647-50, 2019.

Wang, J.; Wang, Q.; Zhu, D.; Jiang, Y. \& Yang, S. Localization of the center of the intramuscular nerve dense region of the medial femoral muscles and the significance for blocking spasticity. Ann. Anat., 231:151529, 2020.

Zhang, G. L.; Meng, H.; Huang, J. H.; Hong, X. F.; Zhang, H. S.; Liu, X. T.; Luo, H. Y. \& Liu, W. Y. Reconstruction of digital skin defects with the free wrist crease flap. J. Reconstr. Microsurg., 31(6):471-6, 2015.

\section{Corresponding author: \\ Shengbo Yang \\ Department of Anatomy \\ Zunyi Medical University \\ 6 West Xufu Road \\ Xinpu Developing Zones \\ Zunyi 563099 \\ CHINA}

\section{Email: yangshengbo8205486@163.com}

Received: 04-11-2020

Accepted: 23-12-2020 